\title{
High Pressure Band Structure, Metallization and Superconductivity of $\mathrm{ZnSe}$ and CdSe
}

\author{
C. Nirmala Louis ${ }^{1}$, A. Amalraj ${ }^{2, *}$ \\ ${ }^{1}$ Assistant Professor of Physics, Holy Cross College, Nagercoil, Tamil Nadu, India \\ ${ }^{2}$ Professor in Chemistry, St. Jerome's College, Anandhanadarkudy, KK District, Tamil Nadu, India \\ *Corresponding Author: arajambrose@yahoo.co.in
}

Copyright $@ 2013$ Horizon Research Publishing All rights reserved.

\begin{abstract}
The high pressure band structure, density of states, metallization, structural phase transition and superconductivity of cubic zinc blende type zinc selenide $(\mathrm{ZnSe})$ and cadmium selenide (CdSe) are investigated using the full potential linear muffin-tin orbital (FP-LMTO) method. The ground state properties and band gap values are compared with the experimental results. The equilibrium lattice constant, bulk modulus and its pressure derivetive and the phase transition pressure at which the compounds undergo structural phase transition from $\mathrm{ZnS}$ to $\mathrm{NaCl}$ are predicted from the total energy calculations. The density of states at the Fermi level $\left(N\left(E_{\mathrm{F}}\right)\right)$ gets enhanced after metallization, which leads to the superconductivity in $\mathrm{ZnSe}$ and CdSe. The superconducting transition temperatures $(T c)$ of $\mathrm{ZnSe}$ and $\mathrm{CdSe}$ are obtained as a function of pressure for $\mathrm{NaCl}$ structure. $\mathrm{ZnSe}$ and $\mathrm{CdSe}$ come under the class of pressure induced superconductors. When pressure is increased $T c$ increases in both compounds. The dependence of $T c$ on electron - phonon mass enhancement factor $\lambda$ shows that $\mathrm{ZnSe}$ and $\mathrm{CdSe}$ are electron-phonon-mediated superconductors.
\end{abstract}

Keywords Metallization, Superconductivity, Znse and Cdse, High Pressure

Subject Classification: $71,71.15 \mathrm{Fv}, 61.50 . \mathrm{Ks}$, 64.70.Kb, 74.62.Fj

\section{Introduction}

The physical properties of materials undergo a variety of changes when they are subjected to high pressure ${ }^{[1]}$. The increase of pressure means the significant decrease in volume, which results in the change of electronic states and crystal structure. With the development of high pressure experimental techniques, investigations on pressure-induced structural phase transition, semiconductor-metal transition and superconducting transition are getting the attention of all. In particular, there is a great interest in the pressure induced structural phase transition and metallization of the group
II-VI compounds $\mathrm{ZnSe}$ and CdSe. One of the most important characteristics of semiconductor, which distinguish it from the metals and insulators, is its energy band gap. This property determines among other things, the wavelength of light which it can absorb or emit. As a result of this, wide range of LEDs and Lasers can be constructed with the wide range of visible and infrared portion of the spectrum. Concentration of impurities or doping materials also play important role to control the electronic and optical properties of semiconductors ${ }^{[1]}$.

Group II-VI compounds are a wide band gap semiconductors with a range of technological applications including electronic and electro-optic devices, catalysis, chemical sensors and conductive solar cell window layers ${ }^{[2,3]}$. Hence, to gain a fundamental understanding of the structural phase transition, metallization and superconductivity of $\mathrm{ZnSe}$ and $\mathrm{CdSe}$, the electronic band structure studies of these materials are essential and this motivated the present investigation. The electronic configuration of $\mathrm{Zn}, \mathrm{Cd}$ and $\mathrm{Se}$ are $[\mathrm{Ar}] 3 d^{10} 4 s^{2}(\mathrm{Z}=30)$, $[\mathrm{Kr}] 4 d^{10} 5 s^{2}(\mathrm{Z}=48)$ and $[\mathrm{Ar}] 3 d^{10} 4 s^{2} 4 \mathrm{p}^{4}(\mathrm{Z}=34)$ respectively. The valence electronic configurations chosen in our calculation are $3 d^{10} 4 s^{2}$ for $\mathrm{Zn}, 4 d^{10} 5 s^{2}$ for $\mathrm{Cd}$ and $4 s^{2}$ $4 p^{4}$ for $\mathrm{Se}$. The band structures and density of states corresponding to various pressures are obtained using the full potential linear muffin-tin orbital (FP-LMTO) method ${ }^{[4]}$. The calculated total energies were fitted to the Murnaghan's equation of state (EOS) ${ }^{[3]}$, to determine the phase transition pressure and other ground state properties. Generally selenide compounds ( $\mathrm{ZnSe}$ and $\mathrm{CdSe}$ ) crystallize in zinc blende structure $(\mathrm{ZnS})^{[1,5]}$. In our calculation we have chosen zinc blende structure as the normal pressure structure of these compounds. Subjecting $\mathrm{ZnSe}$ and $\mathrm{CdSe}$ to high pressure leads to pressure induced metallization, structural phase transition and superconducting transition.

The band gap value for $\mathrm{ZnSe}$ and $\mathrm{CdSe}$ were experimentally measured by Goni et al ${ }^{[6]}$. Theoretically numerous results on the band structure of $\mathrm{ZnSe}$ and $\mathrm{CdSe}$ exist ${ }^{[3,7-14]}$. Recently, Chunyuanet al ${ }^{[1]}$ presented the electrical property and phase transition of CdSe under high pressure. Also Shchennikov etal [2] presented thermoelectric 
properties and phase transitions of eleven II-VI semiconductors under pressure using Seeback effect.There is no high pressure studies related to metallization and superconductivity in both $\mathrm{ZnSe}$ and $\mathrm{CdSe}$. This motivated us to take up the present investigation of these compounds under high pressure. In this work, full potential linearized muffin tin orbital method (FP-LMTO) is employed to study the effect of pressure on the band structure of these compounds.

All solids would become superconductors under sufficiently high pressure ${ }^{[16]}$. The non-metallic compounds with ionic or covalent bonding also become metals and subsequently start superconducting under pressure. Experimentally it is found that, the ionic compound CsI is a metal under high pressure and as the pressure is increased, superconductivity sets in CsI ${ }^{[17]}$. These results lead us to expect superconductivity in other ionic and semiconducting compounds under high pressure; especially those compounds which have already become metals. Prior to metallization these compounds undergo structural phase transition from zinc blende to $\mathrm{NaCl}$ structure. We have analyzed the phenomena of metallization and superconductivity at high pressure $(\mathrm{NaCl})$ structures of these materials. It is hoped that this analysis will enable us to make some general statement regarding the path to high $T c$ superconductivity in covalent compounds.

In Section 2, we give the details of the calculational procedure and electronic band structure corresponding to various pressures. The ground-state properties, structural phase transition, metallization and superconducting transition temperature $T \mathrm{c}$ and its variation under pressure are discussed in Section 3. Concluding remarks are given in Section 4

\section{Band Structure and Density of States}

\subsection{Calculational Procedure}

The electronic band structure calculations were performed for $\mathrm{ZnSe}$ and $\mathrm{CdSe}$ corresponding to different reduced volumes both in $\mathrm{ZnS}$ and $\mathrm{NaCl}$ structures, by the first-principle linear muffin-tin orbital (FP-LMTO) method. We have used FP-LMTO method with in generalized gradient approximation (GGA) ${ }^{[18]}$. We have used the exchange-correlation potential of Ceperley and Alder as parametrized by Perdew and Zunger [19]. We give here only the calculational details. The electronic configuration of $\mathrm{Zn}$, $\mathrm{Cd}$ and Se are $[\mathrm{Ar}] 3 d^{10} 4 s^{2}(\mathrm{Z}=30),[\mathrm{Kr}] 4 d^{10} 5 s^{2}(\mathrm{Z}=48)$ and $[\mathrm{Ar}] 3 d^{10} 4 s^{2} 4 \mathrm{p}^{4}(Z=34)$ respectively. The valence electronic configurations chosen in our calculation are $3 d^{10}$ $4 s^{2}$ for $\mathrm{Zn}, 4 d^{10} 5 s^{2}$ for $\mathrm{Cd}$ and $4 s^{2} 4 p^{4}$ for Se. There are 18 valence electrons contributing to the valence bands [20]. The final energy convergence is within $10^{-5} \mathrm{Ry}$. The calculated total energies were fitted to Murnaghan's equation of state (EOS) [21], to determine the pressure derivative of bulk modulus $\mathrm{B}_{\mathrm{o}}$ '.

\subsection{Band Structure and Density of States of Znse and Cdse under Pressure}

The band structures and density of states of $\mathrm{ZnSe}$ and CdSe were computed for various reduced volumes ranging from $V / V_{o}=1.0$ to 0.3 in steps of 0.05 (Figs. 1 to 8). Even though we have obtained the band structure for $V / V_{o}$ values from 1.0 to 0.3 , we have presented here the band structures of $\mathrm{ZnSe}$ and CdSe along the symmetry directions $\Gamma-X-W-L-\Gamma-K$ corresponding to volume compressions $V / V_{o}=1.0$ and $V / V_{o}=0.686$ for $\mathrm{ZnSe}$, and $V / V_{o}=1.0$ and $V / V_{o}=0.6313$ for CdSe only (Figs.1,3,5 and 7). The overall topology of the band structure of $\mathrm{ZnSe}$ and $\mathrm{CdSe}$ at $V / V_{o}=1.0$ (Figs.1 and 5) is same as that of previous calculations ${ }^{[7]}$.

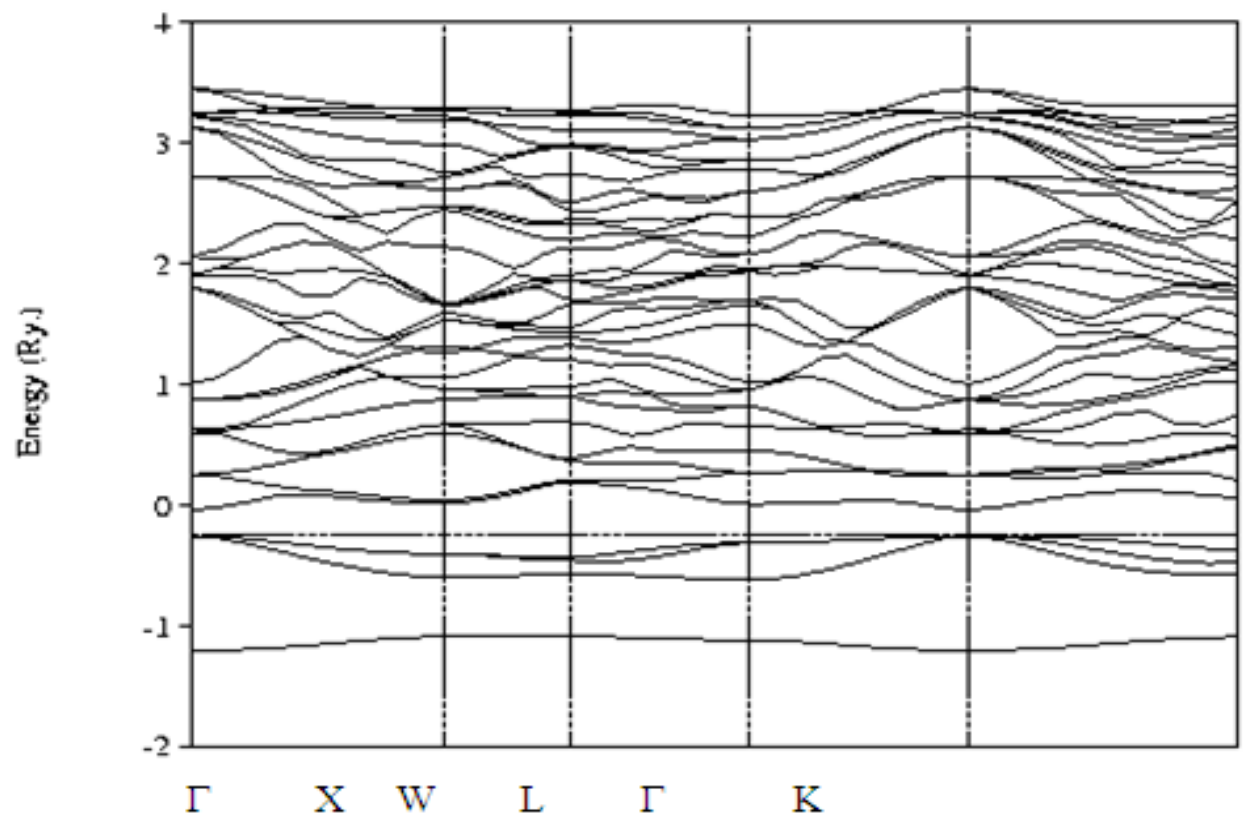

Figure 1. Band structure of $\mathrm{ZnSe}$ at normal pressure 


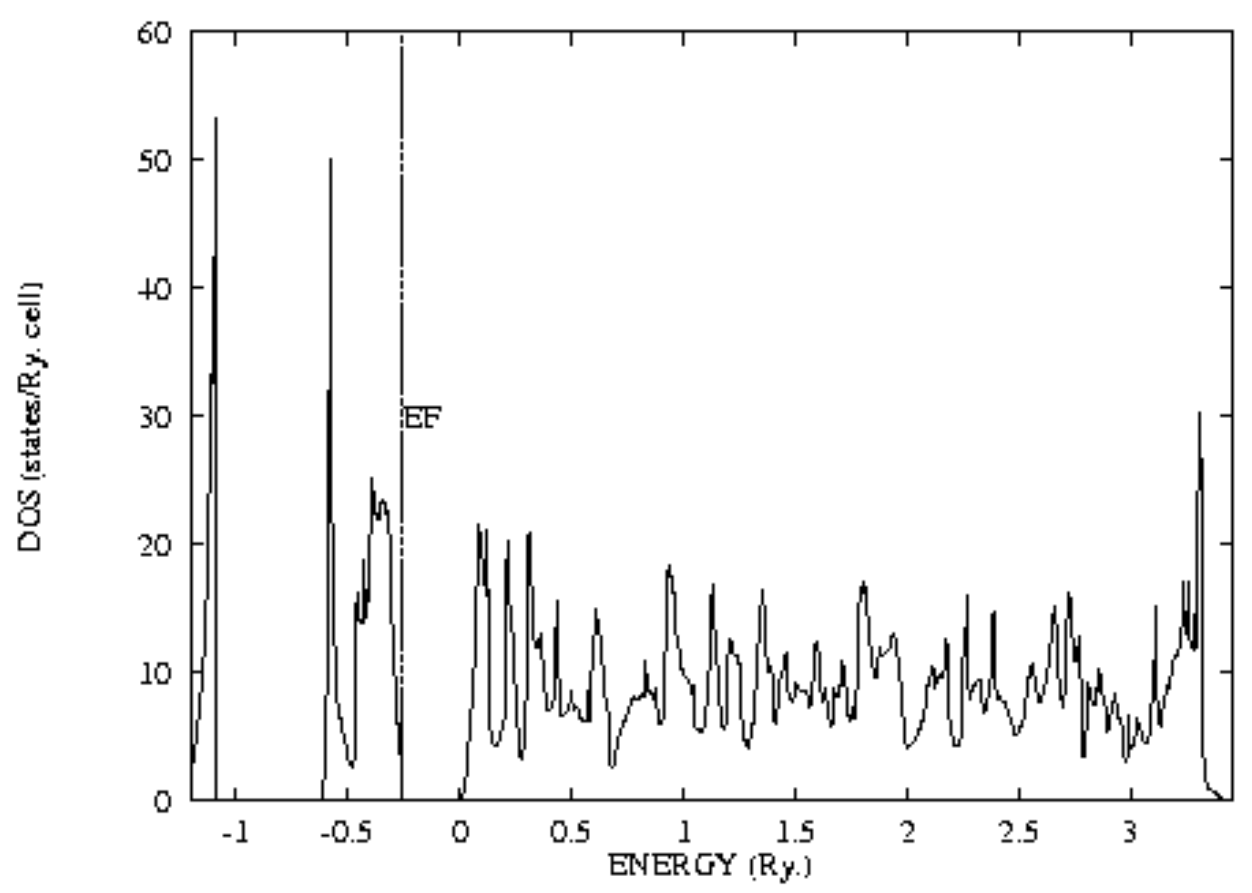

Figure 2. Density of states of $\mathrm{ZnSe}$ at normal pressure

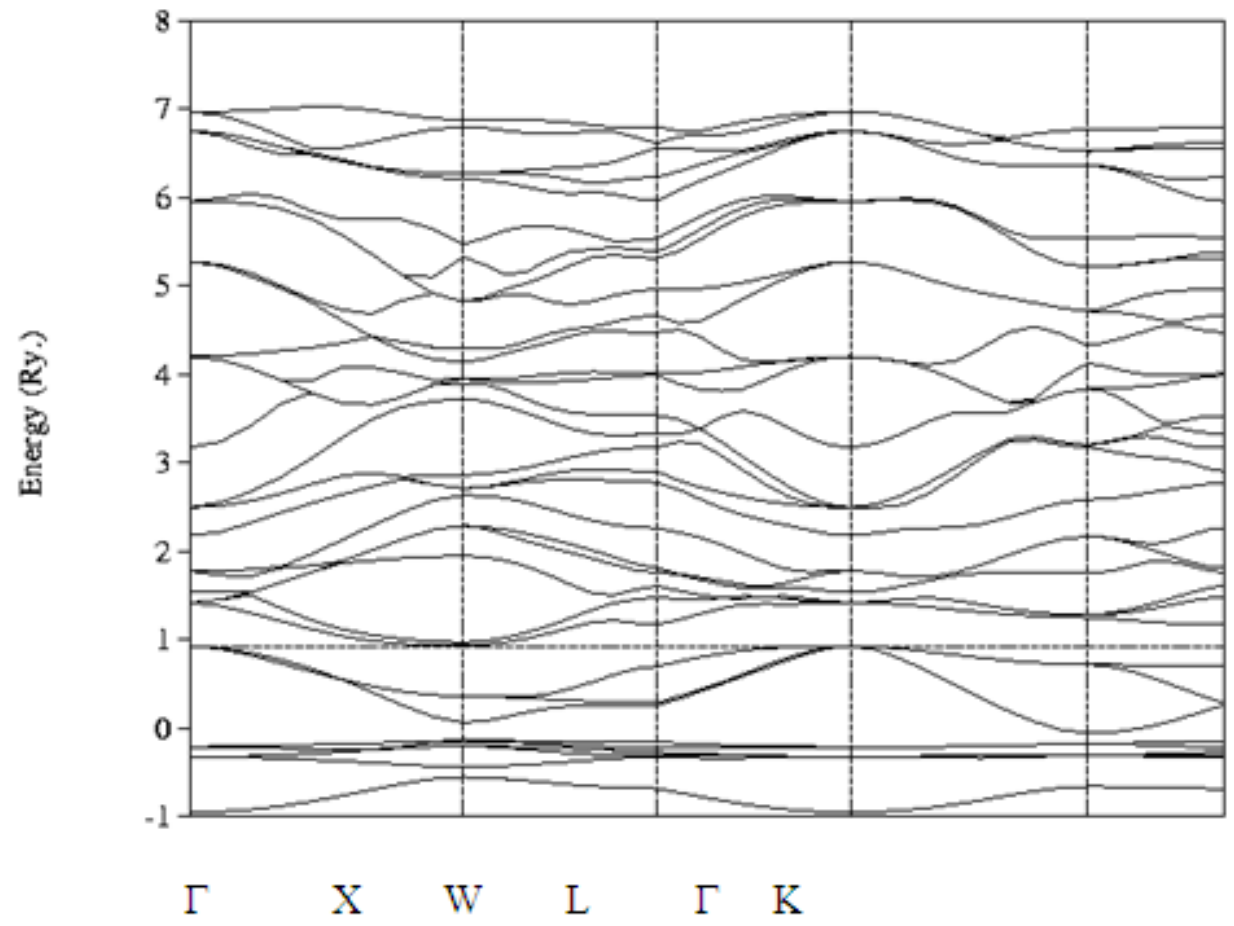

Figure 3. Band structure of $\mathrm{ZnSe}$ at metallization pressure 


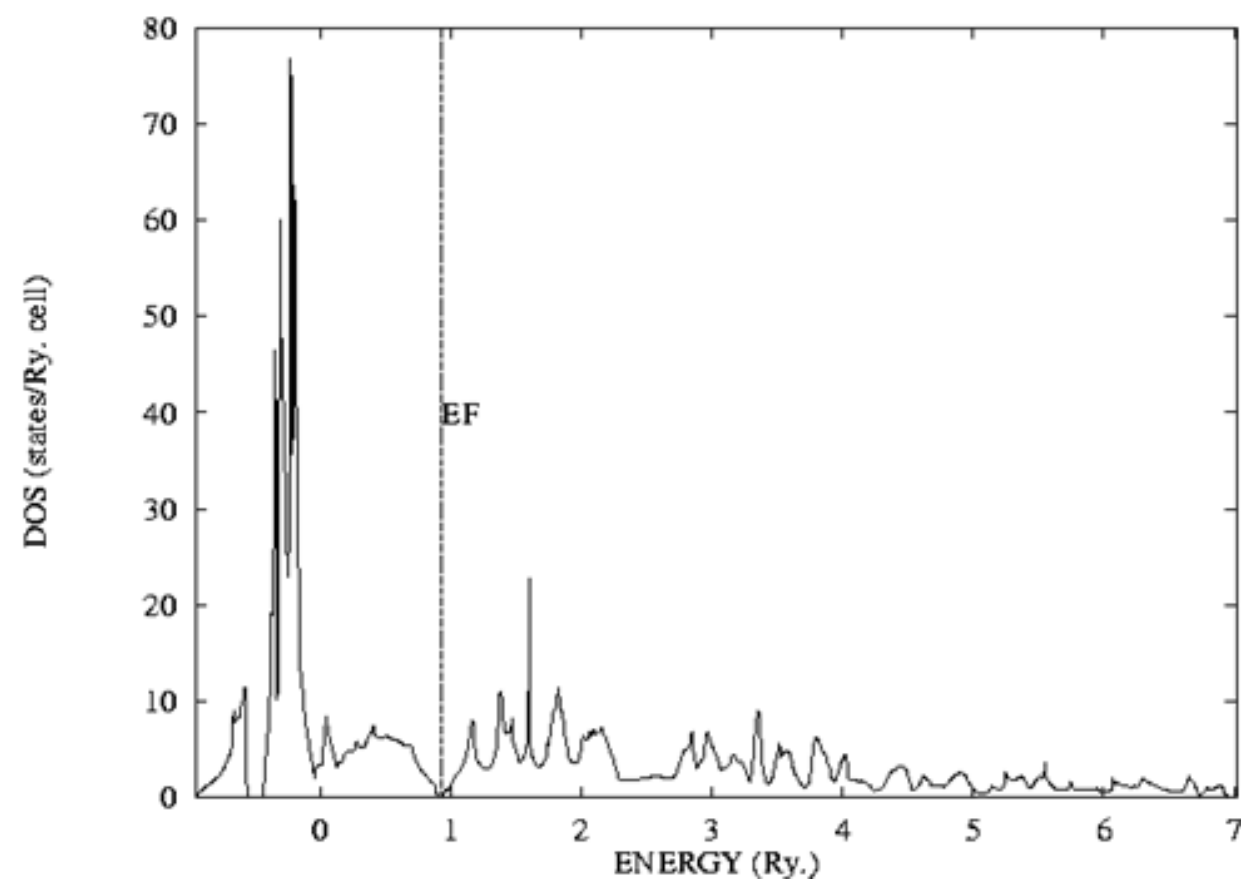

Figure 4. Density of states of $\mathrm{ZnSe}$ at metallization pressure

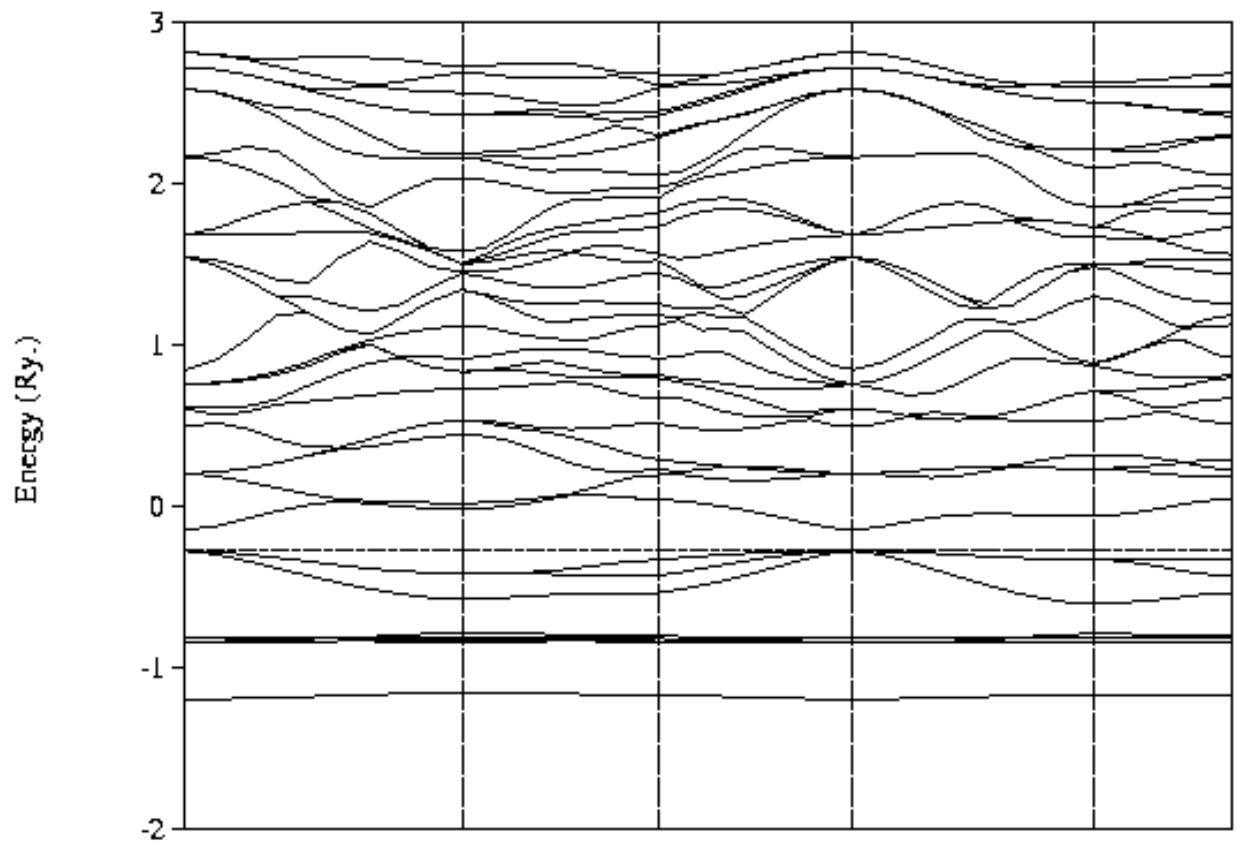

Figure 5. Band structure of CdSe at normal pressure 


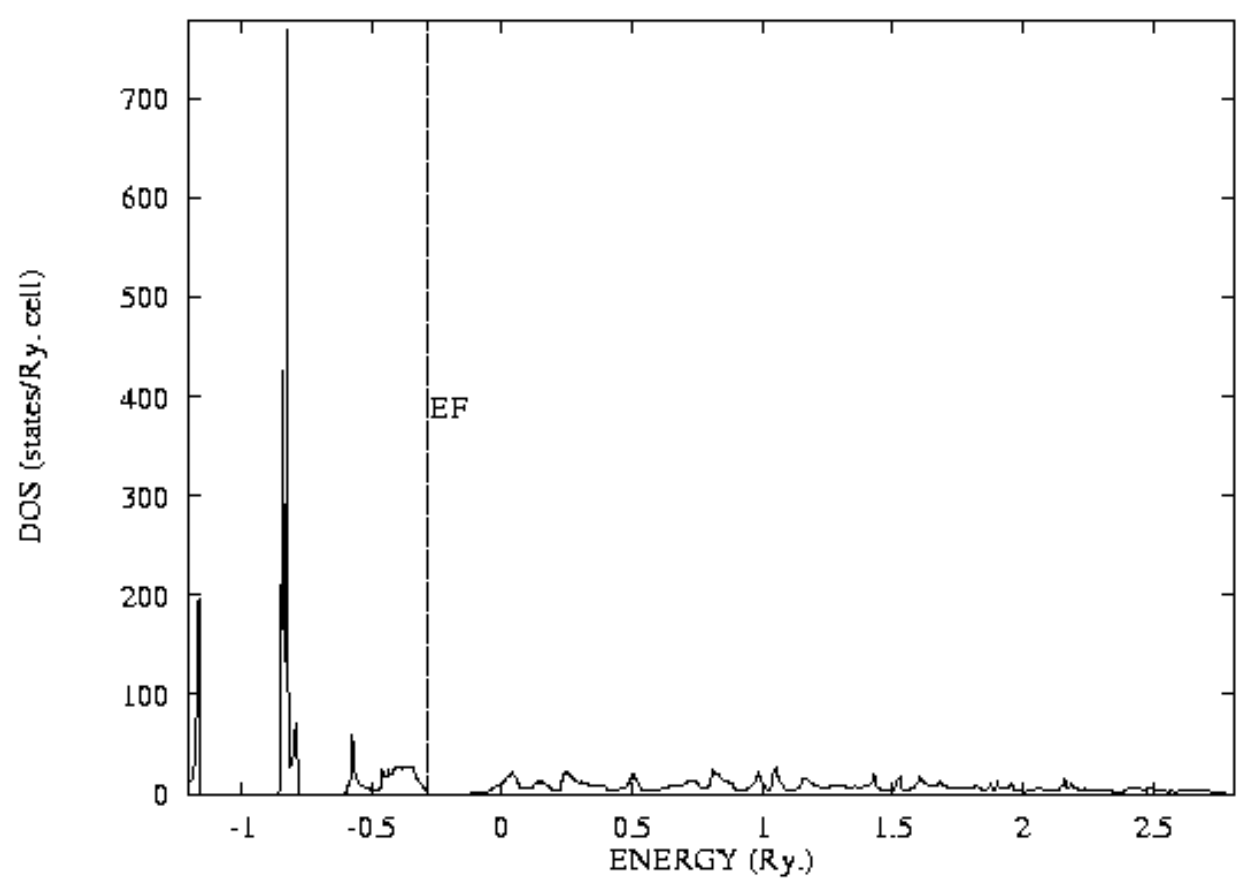

Figure 6. Density of states of CdSe at normal pressure

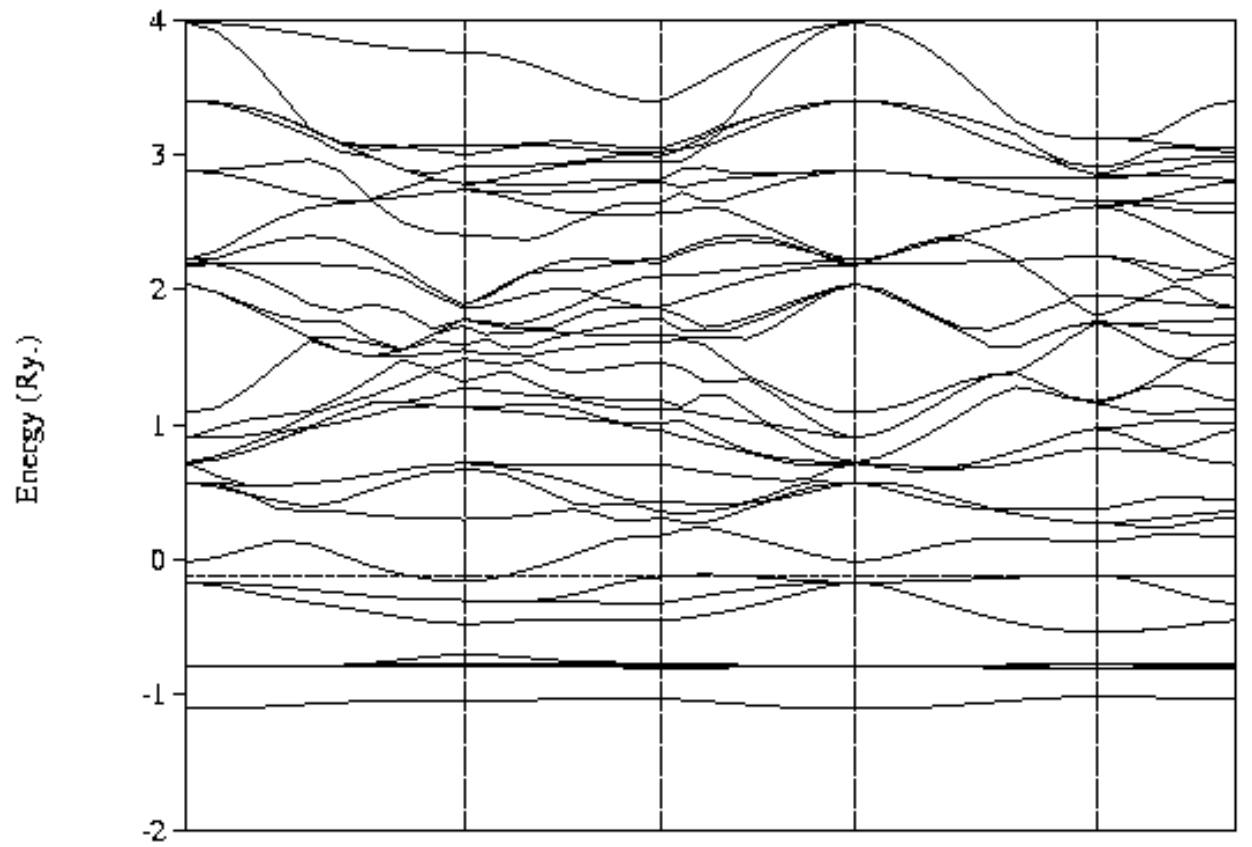

Figure 7. Band structure of $\mathrm{CdSe}$ at metallization pressure 


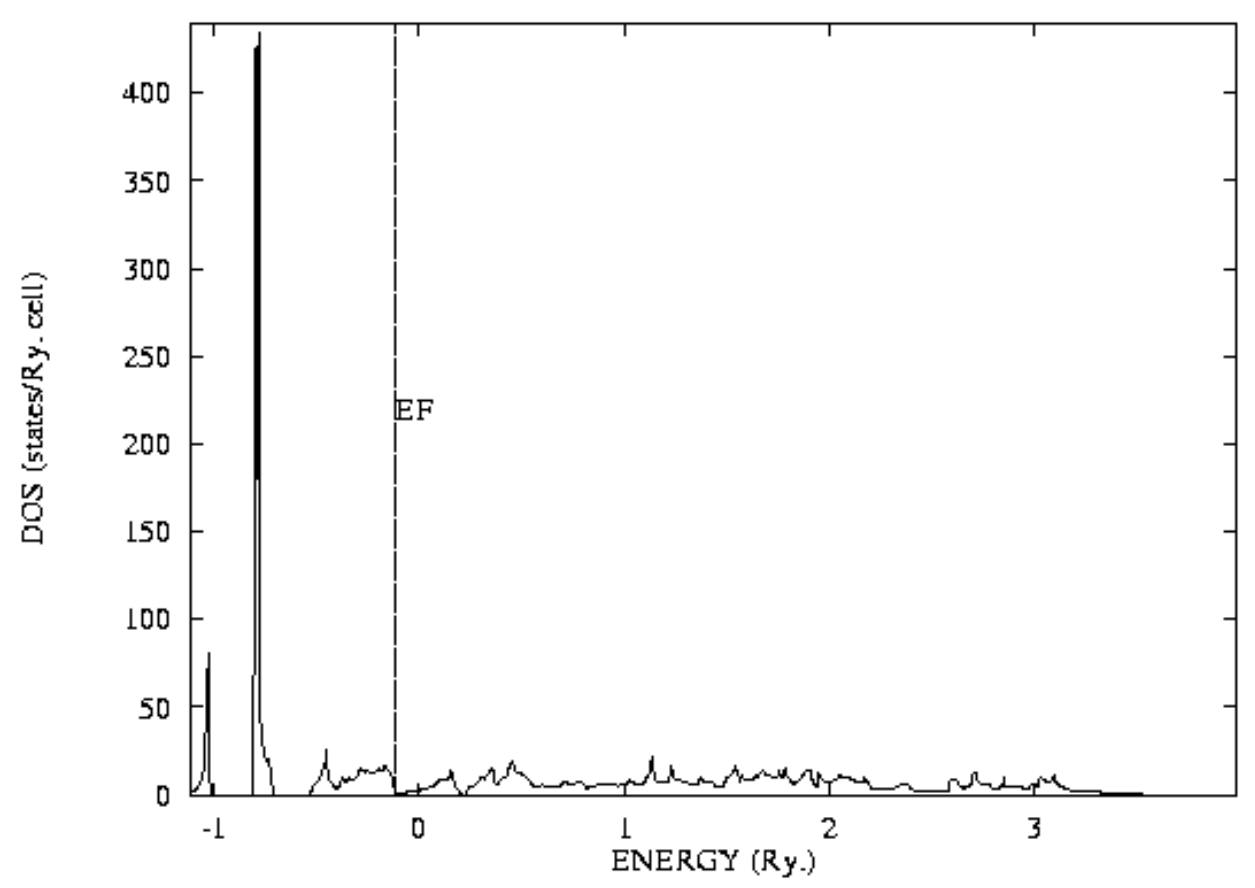

Figure 8. Density of states of CdSe at metallization pressure

A single band which is positioned at the bottom of the valence band arise from $4 \mathrm{~s}^{2}$ electrons of Se (Figs.1 and 5). The three bands appearing below the Fermi level are due to the $4 \mathrm{~s}^{2}$ electrons of $\mathrm{Zn}$ and $4 \mathrm{p}^{4}$ electrons of Se (Fig.1). The empty conduction bands above the Fermi level are due to $4 p$, $4 \mathrm{~d}$ states of $\mathrm{Zn}$ and $4 \mathrm{~d}, 5 \mathrm{~s}$ states of Se (Fig.1). At normal pressure, the direct band gap is found to be $2.67 \mathrm{eV}$ for $\mathrm{ZnSe}$ (at $\Gamma$ point). In Fig. 3 , the five valence bands, which are positioned nearer to the $4 \mathrm{~s}$ bands arise from $4 \mathrm{~d}^{10}$ electrons of Cd (Fig.5). The three bands appearing below the Fermi level are due to the $5 \mathrm{~s}^{2}$ electrons of $\mathrm{Cd}$ and $4 \mathrm{p}^{4}$ electrons of Se. The empty conduction bands above the Fermi level are due to $5 \mathrm{~s}$, $4 \mathrm{~d}$ states of $\mathrm{Se}$ and $5 \mathrm{~d}, 5 \mathrm{p}$ states of $\mathrm{Cd}$ (Fig.5). At normal pressure, the direct band gap is found to be $1.70 \mathrm{eV}$ for $\mathrm{CdSe}$ (at $\Gamma$ point). As pressure is increased, the increase in the width of the valence band and conduction band leads to the narrowing of the band gap and finally metallization in $\mathrm{ZnSe}$ at $\mathrm{V} / \mathrm{Vo}_{0}=0.686\left(\mathrm{P}_{\mathrm{M}}=0.709 \mathrm{Mbar}\right)($ Fig.3 $)$ and $\mathrm{CdSe}$ at $\mathrm{V} / \mathrm{Vo}=0.6313\left(\mathrm{P}_{\mathrm{M}}=0.663\right.$ Mbar) (Fig.7). Metallization occurs via indirect closing of band gap between $\Gamma$ and $X$ points (Figs. 3 and 7). At the metallization pressure, there is appreciable value for density of states at Fermi energy $\mathrm{N}\left(\mathrm{E}_{\mathrm{F}}\right)$. The values of $\mathrm{E}_{\mathrm{F}}$ and $\mathrm{N}\left(\mathrm{E}_{\mathrm{F}}\right)$ are used in studying the pressure variation of superconducting transition temperature.

The density of states (DOS) (states/Ry.) calculations for all the reduced volumes has been carried out but here we have given the DOS histograms of $\mathrm{ZnSe}$ and $\mathrm{CdTe}$ corresponding to normal pressure (Figs.2 and 6) and metallization pressure (Figs. 4 and 8 ). At normal pressure (Fig. 2) the levels arising from $4 \mathrm{~s}^{2}$ electrons of Se give the short spike near the origin. The long spike near the Fermi energy is due to $4 \mathrm{p}^{4}$ and $4 \mathrm{~s}^{2}$ electrons of $\mathrm{Se}$ and $\mathrm{Zn}$ respectively. The short peaks above the Fermi energy $E_{F}$ are due to the $4 p$ and $4 d$ states of $\mathrm{Zn}$ and Se. As the pressure increases the width of the valence band and the empty conduction band get widened. These changes lead to the closing of the band gap under pressure (Fig.4). At normal pressure (Fig. 6) the levels arising from $4 \mathrm{~s}^{2}$ electrons of Se give the short spike near the origin. The longest spikes are due to $4 \mathrm{~d}^{10}$ electrons of $\mathrm{Cd}$. The long spike near the Fermi energy is due to $4 \mathrm{p}^{4}$ and $5 \mathrm{~s}^{2}$ electrons of $\mathrm{Se}$ and $\mathrm{Cd}$ respectively. The short peaks above the Fermi energy $E_{F}$ are due to the $4 \mathrm{p}, 4 \mathrm{~d}, 5 \mathrm{p}$ and $5 \mathrm{~d}$ states of $\mathrm{Cd}$ and $\mathrm{Se}$. As the pressure increases the width of the valence band and the empty conduction band get widened. These changes lead to the closing of the band gap under pressure (Fig.8).

The general features of the band structure and density of states of $\mathrm{ZnSe}$ and $\mathrm{CdSe}$ are similar to that of the alkali halides ${ }^{[23]}$, but in $\mathrm{ZnSe}$ compound $\mathrm{Zn}-3 \mathrm{~d}^{10}$ electrons not involved in the normal pressure band structure and density of states but at high presure band structure and density of states it is involved. In CdSe compound $\mathrm{Cd}-4 \mathrm{~d}^{10}$ electrons involved in the normal and high pressure band structure and density of states. These d electrons play an important role in the band structure ${ }^{[20]}$. The density of states histograms of $\mathrm{ZnSe}$ and $\mathrm{CdSe}$ at high pressure is given in Figs. 4 and 8 respectively. At this pressure $\mathrm{ZnSe}$ and $\mathrm{CdSe}$ are in $\mathrm{NaCl}$ structure. From the histogram, it is seen that the heights of the spikes are considerably reduced when compared to Figs.6 and 8. Reason is when pressure increased $E_{F}$ increases whereas no density of states is available at the Fermi level up to metallization pressure. There are appreciable values for DOS at $V / V_{\mathrm{o}}=0.686$ (Fig. 4) and $V / V_{\mathrm{o}}=0.6313$ (Fig. 8) indicating metallization in $\mathrm{ZnSe}$ and CdSe respectively ${ }^{[1]}$. When pressure increases (reduced volume decreases) the value of the DOS at Fermi energy, $E_{F}\left(N\left(E_{F}\right)\right)$ increases ${ }^{[2]}$. 
The normal pressure DOS trend is changed under high pressure. The increase of pressure leads to the broadening of bands which results in the decrease of density of states value in most of the energy regions and also increases in the width of the valence band and empty conduction bands ${ }^{[24-32]}$. When pressure increases the value of $E_{F}$ increases whereas no density of states is available at the Fermi level up to metallization pressure. Further increase in pressure leads to enhanced density of states at the Fermi level which induces superconductivity ${ }^{[20]}$.

\section{Results and Discussion}

\subsection{Ground State Properties}

The ground state properties and structural phase transitions of $\mathrm{ZnSe}$ and $\mathrm{CdSe}$ are studied from their total energies obtained from our calculation. The total energy is calculated as a function of reduced volume $\left(V / V_{o}\right)$ for both $\mathrm{ZnS}$ and $\mathrm{NaCl}$ phases of $\mathrm{ZnSe}$ and CdSe. Here, $V_{o}$ is the experimental equilibrium volume corresponding to the experimental equilibrium lattice constant. The calculated total energies were fitted to Murnaghan's equation of state [21]

$$
P=1.5 B_{\mathrm{o}}\left[\left(V_{\mathrm{o}} / V\right)^{7 / 3}-\left(V_{\mathrm{o}} / V\right)^{5 / 3}\right]\left[1+0.75\left(B_{\mathrm{o}}{ }^{1}-4\right)\right.
$$

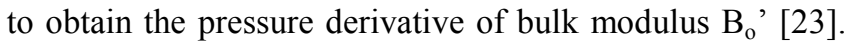
From the variation in the total energy as a function of reduced volume for $\mathrm{ZnSe}$ and $\mathrm{CdSe}$, it is found that, in $\mathrm{ZnSe}$, up to $V / V_{o}=0.942\left(P_{T}=0.1160 \mathrm{Mbar}\right)$ and in CdSe, up to $V / V_{o}=0.967\left(P_{T}=0.0312 \mathrm{Mbar}\right), \mathrm{ZnS}$ structure has the lowest energy and on further reduction of volume $\mathrm{NaCl}$ structure becomes more stable in energy than the $\mathrm{ZnS}$ structure. In Table 1 , the equilibrium lattice constant $\left(a_{\mathrm{o}}\right)$, band gap $\left(E_{\mathrm{g}}\right)$, bulk modulus $\left(B_{\mathrm{o}}\right)$ and its pressure derivative $\left(B_{0}{ }^{1}\right)$ values are compared with experimental and previous theoretical works ${ }^{[1-7]}$. From Table 1, it is observed that one gets larger lattice constant corresponding to smaller bulk modulus. Our estimated bulk modulus of CdSe is less than that of $\mathrm{ZnSe}$, as a result compressibility is higher for $\mathrm{CdSe}$ than $\mathrm{ZnSe}$. Thus, it is noted that the compressibility decreases as the size of the cation decreases. This is the general trend in alkali halides ${ }^{[23]}$, which is confirmed now for $\mathrm{ZnSe}$ and CdSe.

\subsection{Structural Phase Transition}

In our calculation we have chosen the $\mathrm{ZnS}$ structure for both $\mathrm{ZnSe}$ and $\mathrm{CdSe}$ at ambient pressure. The phase stability of the $\mathrm{B} 3(\mathrm{ZnS})$ and $\mathrm{B} 1(\mathrm{NaCl})$ structures of $\mathrm{ZnSe}$ and $\mathrm{CdSe}$ is analysed using the enthalpy calculation ${ }^{[23]}$. The enthalpy $H(P)$ is defined by

$$
\mathrm{H}(\mathrm{P})=\mathrm{E}_{\mathrm{tot}}(\mathrm{P})+\mathrm{PV}(\mathrm{P})
$$

and the transition pressure corresponding to the phase transition from $\mathrm{B} 3$ to $\mathrm{B} 1$ is obtained from the relation

$$
H_{B 3}(P)=H_{\mathrm{B} 1}(P)
$$

where $H_{\mathrm{B} 3}$ and $H_{\mathrm{B} 1}$ are the enthalpies of the $\mathrm{B} 3$ and $\mathrm{B} 1$ phases respectively. From the enthalpy calculation, the phase transition pressure $\left(\mathrm{P}_{\mathrm{T}}=0.1160 \mathrm{Mbar}\right)$ and the corresponding reduced volume $\left(\left(\mathrm{V} / \mathrm{V}_{\mathrm{o}}\right)_{\mathrm{T}}=0.942\right)$ are estimated for $\mathrm{ZnSe}$ and the phase transition pressure $\left(P_{T}=0.0312 \mathrm{Mbar}\right)$ and the corresponding reduced volume $\left(\left(\mathrm{V} / \mathrm{V}_{\mathrm{o}}\right)_{\mathrm{T}}=0.967\right)$ are estimated forCdSe (Table.2).

Our calculated phase transition pressure of $\mathrm{ZnSe}$ supports the experimental value of $\left.0.125 \mathrm{Mbar}^{[31]}\right)$. In the case of $\mathrm{CdSe}$, experimental value is $0.032 \mathrm{Mbar}^{[32]}$. The mechanism for the phase transition is a geometric effect involving a change in the coordination number from 4 in the $\mathrm{ZnS}$ phase to 6 in the $\mathrm{NaCl}$ phase ${ }^{[20]}$.

\subsection{Metallization}

At normal pressure, $\mathrm{ZnSe}$ and $\mathrm{CdSe}$ are direct band gap semiconductors. With the increase of the pressure, the band gap decreases and at a particular pressure, there is a closing of the band gap. The band structure corresponding to metallization of $\mathrm{ZnSe}$ and CdSe are shown in Figs. 3 and 7 respectively. In $\mathrm{ZnSe}$ and $\mathrm{CdSe}$, the metallization occurs through indirect closing of the band gap between $\Gamma$ and $X$ points (Figs.3and 7). The metallization volume of $\mathrm{ZnSe}$ is $V / V_{o}=0.686(\mathrm{NaCl}$ structure $)$, which corresponds to the pressure $P_{M}=0.709 \mathrm{Mbar}$. In the case of $\mathrm{CdSe}$, the indirect closure of the band gap between $\Gamma$ and $X$ point takes place at the reduced volume $V / V_{o}=0.6313(\mathrm{NaCl}$ structure), the corresponding metallization pressure is $0.663 \mathrm{Mbar}$ (Fig.7). This value is good agreement with the experimental value of $0.7 \mathrm{Mbar}^{[1]}$. For $\mathrm{ZnSe}$, no experimental metallization value available for comparison.

The metallization pressure calculated for $\mathrm{ZnSe}$ is greater than that of CdSe (Table 2). This means, in group II-VI compounds, metallization pressure increases with the decreasing size of the group - II element. This trend is similar to the trend observed in alkali halides having $\mathrm{NaCl}$ structure at ambient conditions (metallization pressure increases with decreasing size of alkali metal ${ }^{[23]}$ ). The reason for this similarity is that, prior to metallization, alkali halides undergo structural transition from $\mathrm{NaCl}$ to $\mathrm{CsCl}$ structure whereas group II-VI compounds undergo structural transition from $\mathrm{ZnS}$ to $\mathrm{NaCl}$ structure ${ }^{[7]}$. At the metallization pressures, the values for density of states at Fermi energy $N\left(E_{\mathrm{F}}\right)$ are very small, which indicate that metallization has just set in $\mathrm{ZnSe}$ and CdSe ( Figs. 4 and 8 respectively).

Thereafter $N\left(E_{\mathrm{F}}\right)$ increases slowly with pressure and becomes fairly large at a particular value of $V / V_{0}$. The values of $E_{\mathrm{F}}$ and $N\left(E_{\mathrm{F}}\right)$ corresponding to different $V / V_{\mathrm{o}}$ are used in studying the pressure variation of superconducting transition temperature ${ }^{[20] .}$ However, there is no experimental or theoretical study available for comparison at these pressures. 
Table 1. Equilibrium lattice constant $\left(a_{o}\right)$, bulk modulus $\left(B_{o}\right)$ and its pressure derivative $\left(B_{o}{ }^{l}\right)$ of $\mathrm{ZnSe}$ and CdSe in $\mathrm{ZnS}$ structure

\begin{tabular}{|c|c|c|c|c|c|c|}
\hline \multirow{2}{*}{$\begin{array}{l}\text { Ground } \\
\text { State } \\
\text { Properties }\end{array}$} & \multicolumn{3}{|c|}{$\mathrm{ZnSe}$} & \multicolumn{3}{|c|}{$\mathrm{CdSe}$} \\
\hline & Present work & $\begin{array}{c}\text { Experi } \\
\text { mental works }\end{array}$ & $\begin{array}{l}\text { Previous theoretical } \\
\text { works }\end{array}$ & Present work & $\begin{array}{l}\text { Experimental } \\
\text { works }\end{array}$ & $\begin{array}{l}\text { Previous } \\
\text { theoretical works }\end{array}$ \\
\hline$a_{o} \quad$ a.u. & 10.681 & $10.695[26]$ & $\begin{array}{l}10.421[9] \\
10.432[3]\end{array}$ & 11.441 & $11.455[26]$ & $\begin{array}{l}11.127[9] \\
11.133[3] \\
11.24[32]\end{array}$ \\
\hline$B_{o} \quad$ Mbar & 0.705 & $0.765[26]$ & $\begin{array}{l}0.671[9] \\
0.657[3]\end{array}$ & 0.572 & $0.585[26]$ & $\begin{array}{c}0.519[9] \\
0.417[3] \\
0.515[27] \\
\end{array}$ \\
\hline$B_{o}{ }^{l}$ & 5.201 & $5.53[8]$ & 4.657 [3] & 4.681 & $4.697[8]$ & $4.543[3]$ \\
\hline $\begin{array}{l}E_{g} \\
\mathrm{eV}\end{array}$ & 2.67 & $2.71[6]$ & $\begin{array}{l}1.820[7] \\
1.754[3] \\
\end{array}$ & 1.70 & 1.751 & $\begin{array}{l}1.360[7] \\
1.192[3] \\
\end{array}$ \\
\hline
\end{tabular}

Table 2. Metallization, phase transition and superconducting transition pressures in $\mathrm{ZnSe}$ and CdSe

\begin{tabular}{|c|c|c|c|c|c|c|}
\hline \multirow{2}{*}{$\begin{array}{l}\text { Gallium } \\
\text { Compound }\end{array}$} & \multicolumn{2}{|c|}{ Metallization } & \multicolumn{2}{|c|}{ Phase transition } & \multicolumn{2}{|c|}{$\begin{array}{l}\text { Onset of superconducting } \\
\text { transition }\end{array}$} \\
\hline & $\begin{array}{c}P_{\mathrm{M}} \\
\mathrm{Mbar}\end{array}$ & $\left(V / V_{o}\right)_{\mathrm{M}}$ & $\begin{array}{c}P_{\mathrm{T}} \\
\text { Mbar }\end{array}$ & $\left(V / V_{o}\right)_{\mathrm{T}}$ & $\begin{array}{c}P_{\mathrm{S}} \\
\mathrm{Mbar}\end{array}$ & $\left(V / V_{o}\right) \mathrm{s}$ \\
\hline $\mathrm{ZnSe}$ & 0.709 & 0.6860 & 0.1160 & 0.942 & 1.5 & 0.475 \\
\hline $\mathrm{CdSe}$ & 0.663 & 0.6313 & 0.0312 & 0.967 & 1.5 & 0.525 \\
\hline
\end{tabular}

\subsection{Superconductivity in ZnSe and CdSe under Pressure}

The promotion of $s$ electron to $d$ shell in solids is one of the factors which will induce superconductivity. Under very high pressure, selenides compounds are not only metals but also superconductors. The theory of Gaspari and Gyorffy ${ }^{\text {[33] }}$ in conjunction with McMillan's formula is used to calculate Tc.

The electron - phonon mass enhancement factor, $\lambda$ is ${ }^{[33]}$

$$
\lambda=\frac{N\left(E_{F}\right)\left\langle I^{2}\right\rangle}{M\left\langle\omega^{2}\right\rangle}
$$

where $M$ is the atomic mass, $\left\langle\omega^{2}\right\rangle$ is an average of the phonon frequency square and $\left\langle I^{2}\right\rangle$ is an average (over the Fermi energy) of the electron - phonon matrix element square ${ }^{[34]}$. $\left\langle I^{2}\right\rangle$ (in Rydbergs) can be written as

$$
\left\langle I^{2}\right\rangle=2 \sum_{l} \frac{(l+1)}{(2 l+1)(2 l+3)} M_{l, l+1}^{2} \frac{N_{l}\left(E_{F}\right) N_{l+1}\left(E_{F}\right)}{N\left(E_{F}\right) N\left(E_{F}\right)}
$$

Where

$$
M_{l, l+l}=-\phi_{l} \phi_{l+l}\left[\left(\mathrm{D}_{\mathrm{l}}\left(\mathrm{E}_{\mathrm{F}}\right)-1\right)\left(\mathrm{D}_{1+1}\left(\mathrm{E}_{\mathrm{F}}\right)+1+2\right)+\left(\mathrm{E}_{\mathrm{F}}-\mathrm{V}(\mathrm{S})\right) \mathrm{S}^{2}\right]
$$

and in this,

$\phi_{l}$ is the radial wave function at the muffin-tin sphere radius corresponding to the Fermi energy.

$\mathrm{D}_{1}$ is the logarithmic derivative of the radial wave function at the sphere boundary.

$\mathrm{V}(\mathrm{S})$ is the muffin-tin potential at the sphere boundary.
$\mathrm{S}$ is the radius of the muffin-tin sphere.

The above quantities are taken from the band structure results. We have calculated $\lambda$ separately for zinc, cadmium and selenide atoms and for the Tc calculation (Eq. (8) ) the mean value of $\lambda$ is used ${ }^{[34]}$.

The average of the phonon frequency square is

$$
\left\langle\omega^{2}\right\rangle=\frac{1}{2} \theta_{D}^{2}
$$

The variation of Debye temperature with pressure $\theta_{D}(P)$ is given by ${ }^{[34]}$

$$
\theta_{D}(P)=\frac{\sqrt{E_{F}}}{\sqrt{E_{F}^{o}}} \frac{a_{o}}{a} \theta_{D}^{o}
$$

where $\theta_{D}^{o}, a_{o}$ and $E_{F}^{O}$ are normal pressure quantities ${ }^{[35]}$. Normal pressure Debye temperature of $\mathrm{ZnSe}$ and CdSe are $271 \mathrm{~K}$ and $220 \mathrm{~K}$ respectively.

The McMillan's formula ${ }^{[36]}$ used for $T c$ calculation,

$$
T c=\frac{\theta_{D}}{1.45} \exp \left[\frac{-1.04(1+\lambda)}{\lambda-\mu^{*}(1+0.62 \lambda)}\right]
$$

gives the good estimate of the Tc value. Here $\mu^{*}$ is the electron-electron interaction parameter which is estimated using the relation ${ }^{[37]}$,

$$
\mu^{*}=\frac{0.26 N\left(E_{F}\right)}{1+N\left(E_{F}\right)}
$$


where $N\left(E_{F}\right)$ is the density of levels per atom per eV at $E_{F}$.

With the results obtained from the self-consistent calculation, we have computed $\theta_{D}, \lambda, \mu^{*}$ and $T c$ as a function of pressure using Eqs.(4 - 9). The onset of superconductivity occurs at $(V / V o)_{s}=0.475$ for $\mathrm{ZnSe}$ and 0.525 for CdSe corresponding to the pressure 1.5 Mbar (Table.2). The calculated values at high pressure $(\mathrm{NaCl})$ structure of $\mathrm{ZnSe}$ and $\mathrm{CdSe}$ are given in Tables. 3 and 4. As pressure increases our computed value of $T c$ increases and reaches a maximum value.

In $\mathrm{ZnSe}$, the highest $\mathrm{Tc}$ obtained in $\mathrm{NaCl}$ structure is 6.627 $\mathrm{K}$ at 3 Mbar (Table. 3). In CdSe, the highest Tc obtained in $\mathrm{NaCl}$ structure is $4.030 \mathrm{~K}$ at $3 \mathrm{Mbar}$ (Table. 4). The calculated $T c$ values depend more sensitively on changes in $\lambda$ than $\mu^{*}$, indicating that these compounds are electron-phonon-mediated superconductors.

Table 3. Variation of $T c$ as a function of pressure for $\mathrm{ZnSe}$ in $\mathrm{NaCl}$ structure

\begin{tabular}{|c|c|c|c|c|}
\hline $\begin{array}{c}\text { Pressure } \\
\mathrm{P}_{\text {Mbar }}\end{array}$ & $\lambda$ & $\begin{array}{c}\theta_{D} \\
\mathrm{~K}\end{array}$ & $\mu^{*}$ & $\begin{array}{c}\mathrm{Tc} \\
\mathrm{K}\end{array}$ \\
\hline 1.5 & 0.242 & 712 & 0.102 & 0.016 \\
\hline 2.0 & 0.354 & 783 & 0.115 & 0.744 \\
\hline 2.5 & 0.412 & 842 & 0.132 & 1.494 \\
\hline 3.0 & 0.564 & 925 & 0.154 & 6.627 \\
\hline
\end{tabular}

Table 4. Variation of $T c$ as a function of pressure for $\mathrm{CdSe}$ in $\mathrm{NaCl}$ structure

\begin{tabular}{|c|c|c|c|c|}
\hline $\begin{array}{c}\text { Pressure } \\
\mathrm{P}_{\text {Mbar }}\end{array}$ & $\lambda$ & $\begin{array}{c}\theta_{D} \\
\mathrm{~K}\end{array}$ & $\mu^{*}$ & $\begin{array}{c}\mathrm{Tc} \\
\mathrm{K}\end{array}$ \\
\hline 1.5 & 0.273 & 656 & 0.118 & 0.025 \\
\hline 2.0 & 0.328 & 721 & 0.124 & 0.220 \\
\hline 2.5 & 0.483 & 796 & 0.135 & 3.646 \\
\hline 3.0 & 0.536 & 858 & 0.162 & 4.030 \\
\hline
\end{tabular}

Table 5. Tc-max values of $\mathrm{ZnSe}$ and $\mathrm{CdSe}$ in high pressure $\mathrm{NaCl}$ structure

\begin{tabular}{|c|c|c|c|c|}
\hline Compound & $\begin{array}{c}a_{o} \\
\text { a.u. }\end{array}$ & $\begin{array}{c}B_{o} \\
\mathrm{Mbar}\end{array}$ & $\begin{array}{c}E_{g} \\
\mathrm{eV}\end{array}$ & $\begin{array}{c}\text { Tc-max } \\
\mathrm{K}\end{array}$ \\
\hline $\mathrm{ZnSe}$ & 10.681 & 0.705 & 2.67 & 6.627 \\
\hline $\mathrm{CdSe}$ & 11.441 & 0.572 & 1.70 & 4.030 \\
\hline
\end{tabular}

Similar to alkali halides and group III-V compounds, group II-VI compounds come under the class of pressure induced superconductors ${ }^{[23,34]}$. When pressure is increased $T c$ increases and reaches the maximum value in high pressure structure. The Tc-max values are given in Table.5 along with the other related parameters corresponding to them. From Table.5, it is noted that the Tc-max values are $6.627 \mathrm{~K}$ and $4.030 \mathrm{~K}$ for $\mathrm{ZnSe}$ and CdSe respectively. When lattice constant decreases Tc-max value increase in Table.5.
This reflects the fact that the structural and band gap configurations play an important role in the superconducting (high Tc-max) behaviour of these compounds under high pressure.

\section{Conclusion}

In the present investigation, the pressure dependent band structures and density of states of $\mathrm{ZnSe}$ and $\mathrm{CdSe}$ are computed and the results are used to study the metallization and superconductivity under high pressure for the first time theoretically. When the pressure is increased there is enhanced overlapping between the wave functions of the neighbouring atoms. As a result the widths of the valence and empty conduction bands increase. These changes lead to the narrowing and closing of band gaps in $\mathrm{ZnSe}$ and $\mathrm{CdSe}$ (metallization). On further increase of pressure, $\mathrm{ZnSe}$ and CdSe become superconductors, and these materials come under the class of electron-phonon-mediated high pressure superconductors. It is also confirmed that the metallization, structural phase transition and onset of superconductivity do not occur simultaneously in these compounds (Table.2).

\section{Acknowledgments}

The financial support from the UGC Minor Research Project - No: MRP-4057/11 (UGC - SERO) dated August 2012 is greatly acknowledged with thanks.

\section{REFERENCES}

[1] Chunyuan He, Chunxiao Gao, Yanzhang Ma, etal, Journal of Physics and Chemistry of Solids 69( issue 9), 2227 (2008).

[2] Vladimir V.Shchennikov and Sergey V. Ovsyannikov, Physica Status solidi(b) 244( issue 1), 437 (2007).

[3] S.Q.Wang and H.Q. Ye, Phys. Rev. B 66, 235111 (2002).

[4] J.Wu, W. Walukiewicz, W. Shan, K.M. Yu, J.W. Ager III, E.E. Haller, H.Lu and W.L. Schaff, Phys.Rev. B 66, 201403 (2002).

[5] D.Fritsch, H. Schmidt and M.Grundmann Phys.Rev.B, 67, 235205,(2003).

[6] A.R.Goni, K.Strossner, K.Syassen and M.Cardona, Phys.Rev. B, 36, 1581 (1987).

[7] Michel Cote, Oleg Zakharov, Angel Rubio and Marvin L.Cohen, Phys.Rev. B, 55 (No.19) 13025 (1997).

[8] M. Levinstein, S. Rumyantsev and M. Shur, Hand book series on semiconductor parameters, vol. 1,2 (London: World Scientific ) 1999.

[9] S. Kalvoda, B.Paulus and P.Fulde, Phys.Rev. B, 55, 4027 (1997).

[10] S.B. Zhang and M.L. Cohen, Phys.Rev. B, 35, 7604 (1987). 
[11] S. Logothetidis, J.Petalas, M.Cardona and T.D.Moustakas, Phys.Rev. B, 50, 18017 (1994).

[12] N.E. Christensen and I. Gorczyca, Phys.Rev. B, 50, 4397 (1994).

[13] M.E. Sherwin and T.J. Drummond, J. Appl. phys. 69, 8423 (1991).

[14] A.F. Wright and J.S. Nelson, Phys.Rev. B, 50, 2159 (1994).

[15] S.Q.Wang and H.Q. Ye, J. Phy. Condensed matter, 14, 9579 (2002).

[16] P.W. Bridgman, Physics of High Pressure, 2nd ed., MacMillan Co., New York (1950).

[17] M. I. Eremets, K. Shimizu, and K. AMAYA, Science 281, 1333 (1998).

[18] O. K. Andersen, O. Jepsen and M.Sob, in Elctronic band structure and its applications, edited by M. Yussouff (Springer, Heidelberg,1987) P.1; S.Limpijumnong and W.L.Lambrecht, Phys.Rev. B, 63, 104103 (2001). Savrasov.S.Yu and Savrasov.D.Yu, Phys. Rev.B,46,12181 (1992).

[19] D.M. Ceperley and B.J. Alder, Phys.Rev .Lett.45 , 566 (1980); J.Perdew and Z. Zunger, Phys.Rev .B23, 5048 (1981).

[20] C. Nirmala Louis, et al Physica Status Solidi (B) 241, (2004) 2489-2500.

[21] F. D. Murnaghan, Proc. Natl. Acad. Sci. USA 30, 244 (1944).

[22] A. Mujica, A. Rubio, A. Munoz and R.J. Needs, Reviews of Modern Physics,75, (2003) 863.

[23] C. Nirmala Louis and K. Iyakutti, Phys. Stat. Sol(b) 236, 614 (2003); Phys. Stat. Sol(b) 233, 339 (2002).
[24] M.L. Cohen and J.R. Chelikowsy, Electronic structure and optical properties of semiconductors, 2nd edition, springer(1989).

[25] A. Lindbaum, S. Heathman, K. Litfin and Y. Meresse, Phys. Rev.B 63, 214101 (2001).

[26] K.H.Hellwege and O.Madelung, Londolt-Bornstein, "Semiconductors, Instrinsic properties of II-VI compounds" New Series group III, Vol.22 (Berlin - Springer) 1982.

[27] J.P.Rino and A.Chatterjee, Phys. Rev.B 65, 195206 (2002).

[28] P.E.Van Camp, V.F. Van Doren and J.T. Dovreese, Sol. Stat. Com. 81, 23 (1992); Phys.Rev. B, 38, 9906 (1988).

[29] Sadao Adachi, “ Propertis of Group IV, III-V, II-VI semiconductors" Wiley series in Materials for electronic and optoelectronics applications.

[30] C. Nirmala Louis and K. Iyakutti, etal J.Phys. Condens.Matter, 16, 1577 (2004);

[31] R.J.Nelmes, M.I.MaMahon, N.G.Wright and D.R.Allan, J.Phys. Chem. Of solids, 56, 545 (1995).

[32] W.C.YU and P.J.Gielisse, Matter.Res.Bull, 6, 621(1971).

[33] G.D. Gaspari and B.L. Gyorffy, Phys .Re v. Lett 29, 801 (1972).

[34] A.AmalRaj and C. Nirmala Louis, J.of theoretical and computational Chemistry, 11,19-33 (2012).

[35] P. Bruesch, Phonons- Theory and Experiments-I, (SpringerVerlag,Berlin, 1982) p.81.

[36] W.L. Mcmillan, Phys. Rev. 167, 331 (1968).

[37] K. H. Bennemann and J.W. Garland, AIP Conf. Proc. 4, 103 (1972). 